\title{
THE GEOMETRY AND THE LAPLACE OPERATOR ON THE EXTERIOR 2-FORMS ON A COMPACT RIEMANNIAN MANIFOLD
}

\author{
GR. TSAGAS AND C. KOCKINOS
}

\begin{abstract}
A compact, orientable, Riemannian manifold of dimension $n$ is considered, with the Laplace operator acting on the exterior 2-forms of the manifold. Examining the spectrum, $\mathrm{Sp}^{2}$, of the Laplace operator acting on 2 -forms, the question is raised whether $\mathrm{Sp}^{2}$ exerts an influence on the geometry of the Riemannian manifold.

To answer this question, after some preliminaries, two compact, orientable, equispectral, i.e., having the same $\mathrm{Sp}^{2}$, Riemannian manifolds are considered in \$3. (We note, in particular, that equispectral implies that the two manifolds are equidimensional.) Assuming further that the second Riemannian manifold has constant sectional curvature, the paper exhibits all the dimensions, commencing with 2, for which the two Riemannian equispectral manifolds have the same constant sectional curvature. In particular, this implies that for certain dimensions, which are explicitly stated, the Euclidean $n$-sphere is completely characterized by the spectrum, $\mathrm{Sp}^{2}$, of the Laplacian on exterior 2 -forms.

Next, two compact, orientable, equispectral, Einsteinian manifolds are considered. (Again, equispectral implies equidimensional.) Assuming that the second Einsteinian manifold is of constant sectional curvature, the paper exhibits all the dimensions for which the two Einsteinian equispectral manifolds have equal constant sectional curvature. In particular, taking the second manifold to be the standard Euclidean sphere, the paper classifies Einsteinian manifolds, which are equispectral to the sphere, by calculating all the dimensions for which the Einsteinian manifold is isometric to the sphere. In short, if one of the Einsteinian manifolds is the sphere, then for certain dimensions, equispectral implies isometric.

In \$4, compact, equispectral, Kählerian manifolds are considered, and additional conditions are examined which determine their geometry. Studying two compact, equispectral, Kählerian manifolds, and again assuming that one of the manifolds is of real, constant, holomorphic, sectional curvature, the paper exhibits all the dimensions for which the two manifolds have equal real, constant, holomorphic, sectional curvatures. As a particular case, the paper classifies all the dimensions for which complex projective space, with Fubini-Study metric, is completely characterized by the spectrum, $\mathrm{Sp}^{2}$, of the Laplacian acting on exterior 2 -forms.
\end{abstract}

The calculations were performed by utilizing an electronic computer.

1. Introduction. Let $(M, g)$ be a compact, orientable, Riemannian manifold of dimension $n$. Let $\Lambda^{k}(M)$ be the set of all exterior $k$-forms on $M$, where

Received by the editors August 12, 1977.

AMS (MOS) subject classifications (1970). Primary 58G99; Secondary 53C20, 53C25, 53C55.

Key words and phrases. Laplace operator, spectrum, geometry of Riemannian, Einsteinian, Kählerian, manifolds, eigenvalues of Laplacian. 
$k=0,1, \ldots, n$. (We also use the notation $k \in[0, n]$.) For $k=0$, we obtain the set $\Lambda^{0}(M)$ of all differentiable functions on $M$.

Let $\Delta=-(d \delta+\delta d)$ be the Laplace operator which acts on the exterior algebra of $M$,

$$
\Lambda(M)=\Lambda^{0}(M) \oplus \Lambda^{1}(M) \oplus \cdots \oplus \Lambda^{n}(M)=\oplus \Lambda^{k}(M) \quad(k \in[0, n])
$$

as follows:

$$
\Delta: \Lambda(M) \rightarrow \Lambda(M), \quad \Delta: \Lambda^{k}(M) \rightarrow \Lambda^{k}(M),
$$

$\Delta: \alpha \mapsto \Delta(\alpha)=-(\delta d+d \delta)(\alpha)=-\delta d(\alpha)-d \delta(\alpha), \forall \alpha \in \Lambda^{k}(M)$.

If the exterior $k$-form is such that $\Delta \alpha=\lambda \alpha$, where $\lambda \in \mathbf{R}$, then $\alpha$ is called a $k$-eigenform, (or simply a $k$-form), and $\lambda$ the eigenvalue associated with $\alpha$. The set of eigenvalues associated with the exterior $k$-forms is called the spectrum of $\Delta$ on $\Lambda^{k}(M)$, and is denoted by $\operatorname{Sp}^{k}(M, g)$. Thus

$$
\begin{aligned}
\operatorname{Sp}^{k}(M, g)=\left\{0 \geqslant \lambda_{1, k}=\cdots=\lambda_{1, k}\right. & >\lambda_{2, k}=\cdots \\
& \left.=\lambda_{2, k}>\lambda_{3, k}>\cdots>-\infty\right\}
\end{aligned}
$$

where each eigenvalue is repeated as many times as its multiplicity, which is finite and the spectrum $\operatorname{Sp}^{k}(M, g)$ is discrete, since $\Delta$ is an elliptic operator.

The spectrum, $\operatorname{Sp}^{k}(M, g)$, exerts an influence on the geometry of $(M, g)$. The purpose of the present paper is to study the relation of $\operatorname{Sp}^{2}(M, g)$ on the geometry of $(M, g)$.

2. Let $(M, g)$ be a compact, orientable, Riemannian manifold of dimension $n$, and consider a chart $(U, \varphi)$ on $M$, so as to obtain a local coordinate system $\left(x^{1}, \ldots, x^{n}\right)$, in terms of which the metric $g$, using the Einstein convention, may be expressed as $g \mid U=g_{i j} d x^{i} d x^{j}$. Let $w$ be an exterior 2-form on $M$. Locally,

$$
\left.w \mid U=w_{i j} d x^{i} \wedge d x^{j} \quad \text { (where } i<j \in[1, n]\right) .
$$

Next, let $\Delta$ be the Laplace operator on the exterior 2-forms $\Lambda^{2}(M)$ on $(M, g)$.

$$
\Delta: \Lambda^{2}(M) \rightarrow \Lambda^{2}(M), \quad \Delta: w \mapsto \Delta(w)
$$

such that the components $\Delta w(U)_{h l}, h<l$, where $h, l \in[1, n]$, are given by

$$
\Delta w(U)_{h l}=-g^{i j} \nabla_{j} \nabla_{i} w_{h l}+w_{h q} E_{l}^{q}+w_{q l} E_{h}^{q}+\frac{1}{2} w_{p q} R_{h l}^{q q}
$$

where $E_{s}^{t}=g^{t v} E_{v s}, R_{h l}^{q q}=g^{t q} g^{v q} R_{t v h l}$. Again, the Einstein convention is used. The components $E_{v s}$ and $R_{t v h l}$ are known.

The spectrum of $\Delta$ for $\Lambda^{2}(M)$ is given

$$
\begin{aligned}
\operatorname{Sp}^{2}(M, g)=\left\{0 \geqslant \lambda_{1,2}=\cdots=\lambda_{1,2}\right. & >\lambda_{2,2}=\cdots \\
& \left.=\lambda_{2,2}>\lambda_{3,2}>\cdots>-\infty\right\} .
\end{aligned}
$$

In order to study the influence of $\operatorname{Sp}^{2}(M, g)$ on the geometry of $(M, g)$, we 
need the Minakshisundarum-Pleijel-Gaffney asymptotic expansion given by

$$
\sum_{k=1}^{\infty} e^{\lambda_{k, 2} t} \sim \sum_{\substack{t>0 \\ t \rightarrow 0}}(4 \pi t)^{-n / 2}\left(a_{0,2}+a_{1,2} t+\cdots+a_{q, 2} t^{q}\right)+O\left(t^{q-n / 2}\right)
$$

where $a_{0,2}, a_{1,2}, a_{2,2}, \ldots$ are numbers which can be expressed by

$$
a_{i, 2}=\int_{M} u_{i, 2} d M, \quad i=0,1,2, \ldots,
$$

where $d M$ is the volume element of $M$, and

$$
u_{i, 2}: M \rightarrow \mathbf{R}, \quad i=0,1,2, \ldots,
$$

are functions which are local Riemannian invariants. These can be expressed by the curvature tensor, its associated tensors, and their covariant derivatives.

Some of these have been computed [4],

$$
\begin{gathered}
a_{0,2}=\left(\begin{array}{l}
n \\
2
\end{array}\right) \operatorname{Vol}(M)=n(n-1) / 2 \operatorname{Vol}(M), \\
a_{1,2}=\left(n^{2}-13 n+24\right) / 2 \int_{M} S d M, \\
a_{2,2}=\frac{1}{720} \int_{M}\left[5\left(n^{2}-25 n+120\right) S^{2}\right. \\
-2\left(n^{2}-181 n+1080\right)|E|^{2} \\
\left.+2\left(n^{2}-31 n+240\right)|R|^{2}\right] d M,
\end{gathered}
$$

where $R, E$, and $S$ are the curvature tensor field, the Ricci curvature, and the scalar curvature of $(M, g)$, and $|R|,|E|$, respectively, are the norms of $R, E$, with respect to $g$.

Proposition 2.1. Let $(M, g)$ be a compact, orientable, Riemannian manifold of dimension 2. Then the spectrum $\operatorname{Sp}^{2}(M, g)$ determines the Euler-Poincaré characteristic $\chi(M)$ of $M$.

Proof. Formula (2.2) for $n=2$, gives

$$
a_{1,2}=\int_{M} S d M
$$

Let $\chi(M)$ be the Euler-Poincare characteristic of the two-dimensional manifold $(M, g)$. This is given by the Gauss-Bonnet formula

$$
\chi(M)=\frac{1}{2 \pi} \int_{M} S d M .
$$

From (2.4) and (2.5), we conclude that

$$
\chi(M)=\frac{1}{2 \pi} a_{1,2}
$$

REMARK 2.2. Formula (2.3) for $n=4$, takes the form

$$
a_{2,2}=\frac{1}{360} \int_{M}\left(90 S^{2}-372|E|^{2}+132|R|^{2}\right) d M .
$$


The Euler-Poincaré characteristic $\chi(M)$ of $(M, g)$, for dimension 4, is given by

$$
\chi(M)=\frac{1}{32 \pi^{2}} \int_{M}\left[S^{2}-4|E|^{2}+|R|^{2}\right] d M .
$$

From (2.6) and (2.7), we obtain

$$
360 a_{2,2}-2880 \pi^{2} \chi(M)=\int_{M}\left[-12|E|^{2}+42|R|^{2}\right] d M .
$$

Since, the second member of (2.8) is not a topological invariant, we conclude that $a_{2,2}$ is not a topological invariant.

Problem 2.3. Let $(M, g)$ and $\left(M^{\prime}, g^{\prime}\right)$ be two compact, orientable, Riemannian manifolds. If $\operatorname{Sp}^{2}(M, g)=\operatorname{Sp}^{2}\left(M^{\prime}, g^{\prime}\right)$, is $(M, g)$ isometric to $\left(M^{\prime}, g^{\prime}\right)$ ?

The answer to this problem is negative. This is a consequence of the following counterexample (J. Milnor [3]).

There exist two lattices $L$ and $L^{\prime}$ in $\mathbf{R}^{16}$ such that

$$
\operatorname{Sp}^{0}\left(\mathbf{R}^{16} / L, g_{0} / L\right)=\operatorname{Sp}^{0}\left(\mathbf{R}^{16} / L^{\prime}, g_{0} / L^{\prime}\right)
$$

where $g_{0}$ is the Euclidean metric in $\mathbf{R}^{16}$.

Relation (2.9) implies that

$$
\operatorname{Sp}^{2}\left(\mathbf{R}^{16} / L, g_{0} / L\right)=\operatorname{Sp}^{2}\left(\mathbf{R}^{16} / L^{\prime}, g_{0} / L^{\prime}\right)
$$

But, $\left(\mathbf{R}^{16} / L_{\star} g_{0} / L\right)$ is not isometric to $\left(\mathbf{R}^{16} / L^{\prime}, g_{0} / L^{\prime}\right)$.

From the relation $\operatorname{Sp}^{2}(M, g)=\operatorname{Sp}^{2}\left(M^{\prime}, g^{\prime}\right)$, we conclude that

(i) $\operatorname{dim}(M)=\operatorname{dim}\left(M^{\prime}\right)$,

(ii) $\operatorname{Vol}(M)=\operatorname{Vol}\left(M^{\prime}\right)$,

(iii) $b_{2}(M)=b_{2}\left(M^{\prime}\right)$.

That is, the second Betti numbers are equal, since $b_{2}(M)$ is the multiplicity of 0 in $\operatorname{Sp}^{2}(M, g)$.

3. We consider two compact, orientable, Riemannian manifolds $(M, g)$ and $\left(M^{\prime}, g^{\prime}\right)$, for which we further assume that

$$
\operatorname{Sp}^{2}(M, g)=\operatorname{Sp}^{2}\left(M^{\prime}, g^{\prime}\right)
$$

We study special conditions, which taken together with (3.1), determine the geometry of $(M, g)$.

THEOREM 3.1. Let $(M, g)$ and $\left(M^{\prime}, g^{\prime}\right)$ be two compact, orientable Riemannian manifolds with $\operatorname{Sp}^{2}(M, g)=\operatorname{Sp}^{2}\left(M^{\prime}, g^{\prime}\right)$, (which implies that $\left.\operatorname{dim}(M)=\operatorname{dim}\left(M^{\prime}\right)=n\right)$. If $n=2,3,6,7,14$ or $n \in[17,178]$, then $(M, g)$ is of constant sectional curvature $k$, if and only if $\left(M^{\prime}, g^{\prime}\right)$ is of constant sectional curvature $k^{\prime}$, and $k=k^{\prime}$.

Proof. Let $C, G$ be the Weyl conformal curvature tensor field and the Einstein tensor field, respectively, on $(M, g)$. The components $\left(C_{i j k l}\right)$ and $\left(G_{i j}\right)$ 
of $C$ and $G$, respectively, with respect to $\left(x^{1}, \ldots, x^{n}\right)$ are given by

$$
C_{i j k l}=R_{i j k l}-\alpha\left(E_{j k} g_{i l}-E_{j l} g_{i k}-g_{j k} E_{i l}-g_{i l} E_{i k}\right)+\beta\left(g_{j k} g_{i l}-g_{j l} g_{i k}\right) S,
$$

where $\alpha=1 /(n-1), \beta=1 /(n-1)(n-2)$ and

$$
G_{i j}=E_{i j}-\gamma g_{i j} S \text {, }
$$

where $\gamma=1 / n$.

From (3.2) and (3.3), we obtain

$$
\begin{aligned}
& |C|^{2}=|R|^{2}-4|E|^{2} /(n-2)+2 S^{2} /(n-1)(n-2), \\
& |G|^{2}=|E|^{2}-S^{2} / n .
\end{aligned}
$$

Formula (2.3) by means of (3.4) and (3.5) takes the form

$$
a_{2,2}=\frac{1}{720} \int_{M}\left[Q_{1}|C|^{2}+Q_{2}|G|^{2}+Q_{3} S^{2}\right] d M
$$

where

$$
\begin{aligned}
& Q_{1}=2\left(n^{2}-31 n+240\right) \\
& Q_{2}=\frac{8\left(n^{2}-31 n+240\right)}{n-2}-2\left(n^{2}-181 n+1080\right) \\
& Q_{3}=\frac{4\left(n^{2}-31 n+240\right)}{n(n-1)}-\frac{2\left(n^{2}-181 n+1080\right)}{n}+5\left(n^{2}-25 n+120\right) .
\end{aligned}
$$

By assumption, the Riemannian manifold $\left(M^{\prime}, g^{\prime}\right)$ has constant sectional curvature $k^{\prime}$. Therefore, for $\left(M^{\prime}, g^{\prime}\right)$ we have $C^{\prime}=0, G^{\prime}=0$, and formula (3.6) in this case becomes

$$
a_{2,2}^{\prime}=\frac{1}{720} \int_{M^{\prime}} Q_{3}\left(S^{\prime}\right)^{2} d M^{\prime} .
$$

From (3.1), (3.6), and (3.10), we obtain

$$
\int_{M}\left(Q_{1}|C|^{2}+Q_{2}|G|^{2}+Q_{3} S^{2}\right) d M=\int_{M^{\prime}} Q_{3}\left(S^{\prime}\right)^{2} d M^{\prime}
$$

Now if $n=3,6,7,14$ or $n \in[17,178]$, we have

$$
Q_{1}>0, \quad Q_{2}>0, \quad Q_{3}>0 .
$$

From the relation $a_{1,2}=a_{1,2}^{\prime}$ by means of (2.2); we have

$$
\int_{M} S d M=\int_{M^{\prime}} S^{\prime} d M^{\prime}
$$

which, since $S^{\prime}=$ constant, implies

$$
\int_{M} S^{2} d M \geqslant \int_{M^{\prime}}\left(S^{\prime}\right)^{2} d M^{\prime}
$$

From (3.11), (3.12), and (3.14) we conclude that $|C|^{2}=|G|^{2}=0$, which gives $C=G=0$. Therefore, the Riemannian manifold $(M, g)$ has constant 
sectional curvature $k$. Finally, the relation (3.11) implies $k=k^{\prime}$. The case $n=2$ is obtained from the fact that $\operatorname{Sp}^{2}(M, g)=\operatorname{Sp}^{2}\left(M^{\prime}, g^{\prime}\right)$ implies $\operatorname{Sp}^{0}(M, g)=\operatorname{Sp}^{0}\left(M^{\prime}, g^{\prime}\right)$ and the results in [2]. Q.E.D.

An immediate consequence of the above theorem is the following corollary:

COROllary 3.2. The Euclidean $n$-sphere $\left(S^{n}, g\right)$, for $n=2,3,6,7,14$, or $n \in[17,178]$, is completely characterized by the spectrum of the Laplacian $\Delta$ for exterior 2-forms.

TheOREM 3.3. Let $(M, g)$ and $\left(M^{\prime}, g^{\prime}\right)$ be two compact, orientable, Einsteinian manifolds with $\operatorname{Sp}^{2}(M, g)=\operatorname{Sp}^{2}\left(M^{\prime}, g^{\prime}\right)$, (which implies that $\operatorname{dim}(M)=$ $\left.\operatorname{dim}\left(M^{\prime}\right)=n\right)$. If $n \in[2,7], n=14$, or $n \geqslant 17$, and $\left(M^{\prime}, g^{\prime}\right)$ has constant sectional curvature $k^{\prime}$, then $(M, g)$ has constant sectional curvature $k$, and $k=k^{\prime}$.

Proof. The assumption that $(M, g)$ is an Einsteinian manifold implies $G=0$, and therefore (3.6) takes the form

$$
a_{2,2}=\frac{1}{720} \int_{M}\left(Q_{1}|C|^{2}+Q_{3} S^{2}\right) d M .
$$

If $n \in[3,7], n=14$, or $n \geqslant 17$, we have

$$
Q_{1}>0, \quad Q_{3}>0
$$

From (3.10), (3.15), and (3.16), we conclude $|C|^{2}=0$, which implies $C=0$. Therefore, the Einstein manifold $(M, g)$ has constant sectional curvature $k$. From (3.14), we have $k=k^{\prime}$.

Comment. The case for $n=2$ follows trivially from the definitions. From the above theorem, we have

COROllary 3.4. Let $(M, g)$ be a compact, orientable, Einstein manifold whose dimension is $n$. If $\operatorname{Sp}^{2}(M, g)=\operatorname{Sp}^{2}\left(S^{n}, g_{0}\right)$, where $\left(S^{n}, g_{0}\right)$ is the standard Euclidean sphere, and $n \in[2,7], n=14$, or $n \geqslant 17$ then $(M, g)$ is isometric to $\left(S^{n}, g_{0}\right)$.

4. Let $(M, g)$ and $\left(M^{\prime}, g^{\prime}\right)$ be two compact, Kählerian manifolds for which we assume that

$$
\operatorname{Sp}^{2}(M, g)=\operatorname{Sp}^{2}\left(M^{\prime}, g^{\prime}\right)
$$

We study special conditions which together with (4.1) determine the geometry on $(M, g)$.

Theorem 4.1. Let $(M, g, J)$ and $\left(M^{\prime}, g^{\prime}, J^{\prime}\right)$ be two compact Kählerian manifolds with $\operatorname{Sp}^{2}(M, g)=\operatorname{Sp}^{2}\left(M^{\prime}, g^{\prime}\right)$. If $n=\operatorname{real} \operatorname{dim}(M)$ then for $n=2$, $6,8,14$ or $n \in\{n=2 m \mid m \in[9,94]\},(M, g, h)$ is of real constant holomorphic sectional curvature $h$, if and only if, $\left(M^{\prime}, g^{\prime}, h^{\prime}\right)$ is of constant holomorphic sectional curvature $h^{\prime}$, and $h=h^{\prime}$.

Proof. We introduce the Bochner curvature tensor field $B$ on $(M, g, J)$, 
whose components $\left(B_{i j k l}\right)$ with respect to the local coordinate system $\left(x^{1}, \ldots, x^{n}\right)$ are given by

$$
\begin{aligned}
B_{i j k l}= & R_{i j k l}-\alpha^{*}\left(E_{j k} g_{i l}-E_{j l} g_{i k}+E_{i l} g_{j k}-E_{i k} g_{j l}+E_{j r} J_{k}^{r} J_{i l}-E_{j r} J_{l}^{r} J_{i k}\right. \\
& \left.+J_{j k} E_{i r} J_{l}^{r}-J_{j l} E_{i r} J_{k}^{r}-2 E_{k r} J_{l}^{r} J_{i j}-2 E_{i r} J_{j}^{r} J_{k l}\right) \\
& +\beta^{*}\left(g_{j k} g_{i l}-g_{j l} g_{i k}+J_{j k} J_{i l}-J_{j l} J_{i k}-2 J_{k l} J_{i j}\right) S
\end{aligned}
$$

where $\alpha^{*}=1 /(n-1)$ and $\beta^{*}=1 /(n+2)(n+4)$.

From (4.2) we obtain the following formula

$$
|B|^{2}=|R|^{2}-16|E|^{2} /(n+4)+8 S^{2} /(n+2)(n+4) .
$$

Formula (2.3) by means of (3.5) and (4.3) takes the form

$$
a_{2,2}=\frac{1}{720} \int_{M}\left(P_{1}|B|^{2}+P_{2}|G|^{2}+P_{3} S^{2}\right) d M
$$

where

$$
\begin{aligned}
& P_{1}=2\left(n^{2}-31 n+240\right) \\
& P_{2}=-2\left(n^{2}-181 n+1080\right)+\frac{32}{(n+4)}\left(n^{2}-31 n+240\right) \\
& P_{3}=5\left(n^{2}-25 n+120\right)-\frac{2}{n}\left(n^{2}-181 n+1080\right)+\frac{16\left(n^{2}-31 n+240\right)}{n(n+2)}
\end{aligned}
$$

If $n=$ real $\operatorname{dim}(M)$, then for $n=6,8,14$ or $n \in\{n=2 m \mid m \in[9,94]\}$, we have

$$
P_{1}>0, \quad P_{2}>0, \quad P_{3}>0 .
$$

If we assume that the Kähler manifold $\left(M^{\prime}, g^{\prime}, J^{\prime}\right)$ has constant holomorphic sectional curvature $h^{\prime}$, then relation (4.4) takes the form

$$
a_{2,2}^{\prime}=\frac{1}{720} \int_{M^{\prime}} P_{3}\left(S^{\prime}\right)^{2} d M^{\prime}
$$

From (4.1), we obtain that $a_{1,2}=a_{1,2}^{\prime}$ implies

$$
\int_{M} S d M=\int_{M^{\prime}} S^{\prime} d M^{\prime}
$$

and that $a_{2,2}=a_{2,2}^{\prime}$ implies

$$
\int_{M}\left(P_{1}|B|^{2}+P_{2}|G|^{2}+P_{3} S^{2}\right) d M=\int_{M^{\prime}} P_{3}\left(S^{\prime}\right)^{2} d M^{\prime} .
$$

Since the holomorphic sectional curvature $h^{\prime}$ of $\left(M^{\prime}, g^{\prime}, J^{\prime}\right)$ is constant, (4.10) implies

$$
\int_{M} S^{2} d M \geqslant \int_{M^{\prime}}\left(S^{\prime}\right)^{2} d M^{\prime}
$$

Relation (4.11) by means of (4.8) and (4.12) gives $|B|^{2}=0$ and $|G|^{2}=0$, which imply $B=0$ and $G=0$. That is, $(M, g, J)$ has constant holomorphic sectional curvature $h$. From (4.10) we obtain $h=h^{\prime}$. The case $n=2$ is 
obtained with the same technique as in Theorem 3.1.

An immediate consequence of the above theorem is the following corollary.

COROllary 4.2. The complex projective space $\left(P^{m}(C), g_{0}, J_{0}\right)$ with FubiniStudy metric $g_{0}$, and real dimension $n$ such that $n=2 m=2,6,8,14$ or $n \in\{n=2 m \mid m \in[9,94]\}$, is completely characterized by the spectrum of the Laplacian on the exterior 2-forms.

ACKnowledgement. Many thanks are due to Raymond E. Orth, Jr. who checked the calculations for $Q_{1}=P_{1}, Q_{2}, Q_{3}, P_{2}, P_{3}$, by running them through a computer from $n=3$ to $n=1000$. Orth found that $Q_{1}=P_{1}$ is always positive, for $n$ integral, except for two zeros which occur at $n=15$ and $n=16 . Q_{2}$ assumes positive values for $n=3$, and $n \in[6,178]$, and becomes negative beginning with 179 . It is also negative at $n=4,5 . Q_{3}$ is positive for $n \in[3,7]$, has a zero at $n=8$, becomes negative for $n \in[9,13]$, and becomes positive again from $n=14$ on.

$P_{2}$ is negative for $n \in[1,5]$, positive for $n \in[6,188]$, and negative for $n$ greater than or equal to $189 . P_{3}$ is negative at $n=1$, positive for $n \in[2,8]$, negative for $n \in[9,13]$, and becomes positive again beginning with $n=14$.

\section{REFERENCES}

1. M. Berger, Sur quelques variétés Riemanniennes compactes d'Einstein, C. R. Acad. Sci. Paris 260 (1965), 1554-1557.

2. M. Berger, P. Gauduchon and E. Mazet, Le spectre d'une variété Riemannienne, Lecture Notes in Math., vol. 194, Springer-Verlag, Berlin and New York, 1971.

3. J. Milnor, Eigenvalues of the Laplace operator on certain manifolds, Proc. Nat. Acad. Sci. U.S.A. 51 (1964), p. 542.

4. V. K. Petodi, Curvature and the fundamental solution of the heat operator, J. Indian Math. Soc. 34 (1970), 269-285.

5. S. Tanno, The spectrum of the Laplacian for 1-forms, Proc. Amer. Math. Soc. 45 (1974), 125-129.

6. Gr. Tsagas, On the spectrum of the Laplacian on the 1-forms on a compact Riemannian manifold, Tensor (to appear).

Department of Mathematics, Faculty of Technology, University of Thessaloniki, Thessaloniki, Greece (Current address of Gr. Tsagas)

SRi international, 333 Ravenswood Avenue, Menlo Park, California 94205

Current address (C. N. Kockinos): 2121 Creeden Avenue, Mountain View, California 94040 